\title{
Éducation pour la santé et pratiques sociales
}

Yannick Jaffré

\section{(2) OpenEdition}

Journals

Édition électronique

URL : http://journals.openedition.org/apad/388

DOI : 10.4000/apad.388

ISSN : 1950-6929

Éditeur

LIT Verlag

Édition imprimée

Date de publication : 15 mars 1992

\section{Référence électronique}

Yannick Jaffré, «Éducation pour la santé et pratiques sociales », Bulletin de l'APAD [En ligne], 3| 1992,

mis en ligne le 06 juillet 2006, consulté le 08 septembre 2020. URL : http://journals.openedition.org/ apad/388; DOl : https://doi.org/10.4000/apad.388

Ce document a été généré automatiquement le 8 septembre 2020

Bulletin de I'APAD 


\title{
Éducation pour la santé et pratiques sociales
}

\author{
Yannick Jaffré
}

1 (Reproduction, avec l'autorisation de l'auteur, de la présentation d'un stage organisé du 31 août au 4 septembre 1992 à Aix-en-Provence par le Ministère français de la Coopération). L'éducation pour la santé n'est souvent décrite qu'en fonction des théories de la communication ("trouver le bon message pour modifier les mauvais comportements"). A l'évidence cette définition ne peut rendre compte ni des nécessaires articulations avec d'autres disciplines (épidémiologie, économie, etc.), ni de la complexité des diverses préventions et des milieux sociaux dans lesquels elle s'exerce. Pour cela nous comprendrons ici l'éducation pour la santé comme une pratique située à l'interface des discours techniques médicaux et les pratiques sociales des populations. Elle a pour tâche d'organiser cette rencontre de manière opérationnelle, en informant les soignants et les populations sur les choix et options des uns et des autres, en améliorant l'accessibilité des structures de santé par une meilleure prise en compte des "demandes" des collectivités locales, en agissant en amont des interventions sanitaires sur les déterminants de santé (alphabétisation, hydraulique, etc.). Comment donc, si l'on accepte cette définition générale, prendre en compte le "public" de la santé publique et mettre en œuvre ces pratiques préventives, socialement innovantes?

2 Travailler dans le cadre de programmes de développement sanitaire implique de posséder de bonnes connaissances techniques professionnelles. Mais, si celles-ci sont nécessaires, elles ne sont pas suffisantes. Toute action s'adressant en effet à des sociétés complexes, définies par de multiples contraintes économiques et des systèmes de valeurs spécifiques, les projets sont l'objet et/ou l'enjeu de multiples interactions entre les techniciens nationaux ou expatriés qui les ont conçus et les diverses populations supposées en être les bénéficiaires.

3 Les populations ne sont ni des ensembles vides de connaissances, ni des communautés trop pleines de croyances. Elles agissent selon diverses logiques, économiques, sociales, symboliques, qu'il importe de connaître et d'analyser pour les comprendre. Si ces 
groupes sociaux n'ont pas toujours raison - il ne s'agit pas ici d'idéaliser "le traditionnel" - il importe de souligner qu'elle n'agissent pas cependant, sans avoir de "bonnes" raisons, définies en fonction de leur système de référence économique et social, pour interpréter et s'approprier les diverses propositions d'innovation que leur proposent les projets de développement.

De la même manière, les personnels de santé ne peuvent être uniquement décrits sous leur aspect de techniciens. Pour être sages-femmes, médecins ou infirmiers, il n'en sont pas moins des personnes agissant en fonction des diverses contraintes que leur impose leur milieu de vie. Dans bien des cas, la résolution des difficultés techniques implique de considérer tout autant les identités sociales de ces divers personnels que leur simple niveau de formation.

5 De ces diverses interactions résulte que, bien souvent, les programmes sanitaires et sociaux ne produisent pas les effets que l'on en attendait. La constatation des écarts entre les objectifs fixés et les effets produits par un projet n'est souvent pas analysée de manière rigoureuse et scientifique mais fait l'objet d'explications psychologiques ("ils ne sont pas motivés"), ou culturalistes ("leur culture ne permet pas de.. ").

6 Comment progresser dans ce domaine ? De même qu'il ne suffit pas d'avoir un cœur pour être cardiologue, le fait de vivre en société ne fait pas le sociologue. Penser l'inscription d'un projet sanitaire dans un ensemble social doit faire l'objet d'un travail élaboré grâce à des connaissances et des méthodologies précises. Ce sont celles-ci que le stage vise à fournir aux participants. 